\title{
Perceptual color difference metric for complex images based on
}

\section{Mahalanobis distance}

\author{
F. H. Imai*, N. Tsumura and Y. Miyake \\ Department of Information and Image Sciences, Chiba University, 1-33 Yayoi-cho, \\ Inage-ku, Chiba-shi, 263-8522 Japan \\ *Munsell Color Science Laboratory, Chester F. Carlson Center for Imaging Science, \\ Rochester Institute of Technology, 54 Lomb Memorial Drive, Rochester, NY, USA \\ e-mail: imai@cis.rit.edu, tsumura@ics.tj.chiba-u.ac.jp, miyake@ics.tj.chiba-u.ac.jp
}

\begin{abstract}
In this paper, a perceptual color difference is presented as an alternative color difference metric for complex images instead of the conventional color difference equations. This color difference is derived based on Mahalanobis distance by using covariance matrices for differences of each color attributes. The covariance matrices for each class of images can be obtained by psychophysical experiments using just noticeable difference in paired comparisons. We compared the resultant matrices for different class of images and the information in the matrix can give very useful trends and clues about which kind of transformation can minimize the perceptual color difference in images when a transformation such as gamut mapping is required.
\end{abstract}


Keywords: color difference for images, Mahalanobis distance, paired comparison, covariance matrix

\section{Introduction}

Considerable work has been accomplished in terms of color difference perception comparing single colored patches. ${ }^{1-10}$ Based on these researches many color difference equations have been proposed. The most widely used color difference equations in the last decades are CIELAB and CIELUV color difference equations recommended in 1976 by the CIE. ${ }^{1}$ In both CIELAB and CIELUV color spaces, the color difference $\Delta E^{*}$ between two arbitrary colors is defined as an Euclidian distance in a uniform space comprising a lightness $L^{*}$ axis and red-green, yellow-blue opponent color axes using rectangular coordinates. The color difference in CIELAB and CIELUV color spaces are given respectively by Equations 1 and 2 .

$$
\begin{aligned}
& \Delta E_{a b}^{\star}=\sqrt{\left(\Delta L^{*}\right)^{2}+\left(\Delta a^{*}\right)^{2}+\left(\Delta b^{\star}\right)^{2}} \\
& \Delta E_{u v}^{*}=\sqrt{\left(\Delta L^{*}\right)^{2}+\left(\Delta u^{\star}\right)^{2}+\left(\Delta v^{*}\right)^{2}}
\end{aligned}
$$

where $a^{*}$ and $b^{*}$ are respectively, the redness-greenness and yellowness-blueness scales in CIELAB color space, and $u^{*}$ and $v^{*}$ are respectively, the redness-greenness and yellowness-blueness scales in CIELUV color space. 
The color difference equation in these spaces can also be expressed using cylindrical coordinates in terms of lightness, chroma and hue. The color difference in cylindrical coordinates in CIELAB and CIELUV are given respectively by equations 3 and 4.

$$
\Delta E_{a b}^{*}=\sqrt{\left(\Delta L^{*}\right)^{2}+\left(\Delta C_{a b}^{*}\right)^{2}+\left(\Delta H_{a b}^{*}\right)^{2}}
$$

where

$$
\Delta H_{a b}^{*}=\sqrt{\left(\Delta E_{a b}^{*}\right)^{2}-\left(\Delta L^{*}\right)^{2}-\left(\Delta C_{a b}^{*}\right)^{2}}
$$

where $\Delta C_{a b}^{*}$ is the chroma difference in CIELAB color space and $\Delta H_{a b}^{*}$ is the hue difference in CIELAB color space.

$$
\Delta E_{u v}^{*}=\sqrt{\left(\Delta L^{*}\right)^{2}+\left(\Delta C_{u v}^{*}\right)^{2}+\left(\Delta H_{u v}^{*}\right)^{2}}
$$

where

$$
\Delta H_{u v}^{*}=\sqrt{\left(\Delta E_{u v}^{*}\right)^{2}-\left(\Delta L^{*}\right)^{2}-\left(\Delta C_{u v}^{*}\right)^{2}}
$$

where $\Delta C_{u v}^{*}$ is the chroma difference in CIELUV color space and $\Delta H_{u v}^{*}$ is the hue difference in CIELUV color space.

In 1976, McLaren was the pioneer in introducing weighting factors for each dimension of color in his color difference equation. ${ }^{2}$ McLaren combined an equation previously published by McDonald that simplifies complex color difference equation ${ }^{3}$ with the use of a weight that gives twice of weight in hue dimension compared to chroma and lightness dimensions, as shown in Equation 5.

$$
\Delta E_{(\mathrm{Mc})^{2}}=\frac{\sqrt{(\Delta L)^{2}+(\Delta C)^{2}+(2 \Delta H)^{2}}}{1+0.02 C}
$$


McDonald also pioneered the use of nonlinear weights in the color difference equation resulting in his $\Delta E_{\mathrm{JPC} 79}$ color difference formula ${ }^{4}$ shown in Equation 6 where $L, C$, and $h$ are calculated from the $\mathrm{ANLAB}^{5} \mathrm{~L}, \mathrm{~A}$, and $\mathrm{B}$ values.

$$
\Delta E_{\mathrm{JPC} 79}=\sqrt{\left(\frac{\Delta L}{S_{L}}\right)^{2}+\left(\frac{\Delta C}{S_{C}}\right)^{2}+\left(\frac{\Delta H}{S_{H}}\right)^{2}}
$$

where $S_{L}$ depends on $L, S_{C}$ depends on $\mathrm{C}$, and $S_{H}$ depends on $C$ and $h$.

ANLAB, or Adams-Nickerson equation was derived in the 1940s using the chromaticvalue theory of color vision and this equation and further development were the basis for CIELAB color difference.

In the last 20 years new color difference formulae have been proposed. In 1984, Clarke, et al. proposed the CMC (1:c) color difference equation. ${ }^{6,7}$ The $\operatorname{CMC}(l: c)$ formula is given in Equation 7. There are weights applied to the difference in lightness, chroma and hue using $S_{L}$ that depends on lightness, $S_{C}$ that depends on chroma and $S_{H}$ that depends on both chroma and hue angle $h$. This equation also has $l$ and $c$ parameters that are chosen according to the material.

$$
\Delta E_{\mathrm{CMCl:C)}}=\sqrt{\left(\frac{\Delta L^{*}}{I S_{L}}\right)^{2}+\left(\frac{\Delta C_{a b}^{*}}{c S_{C}}\right)^{2}+\left(\frac{\Delta H_{a b}^{*}}{S_{H}}\right)^{2}}
$$

Furthermore, Luo and Rigg developed BFD color difference equation $\Delta E_{\mathrm{BFD}(l: c)}$ in 1987 providing a correction for the $\mathrm{CMC}(l: c)$ in the blue region. ${ }^{8,9}$ The BFD color difference equation is shown in Equation 8. 
$\Delta E_{\mathrm{BFD}(l: c)}=\sqrt{\left(\frac{\Delta L_{\mathrm{BFD}}{ }^{*}}{l}\right)^{2}+\left(\frac{\Delta C^{*}}{c D_{C}}\right)^{2}+\left(\frac{\Delta H^{*}}{D_{H}}\right)^{2}+R_{T}\left(\frac{\Delta C^{*} \Delta H^{*}}{D_{C} D_{H}}\right)}$

where $L_{\mathrm{BFD}}=54.6 \log _{0}(Y+1.5)-9.6, D_{C}$ depends on the arithmetic mean value of the compared color 1 and color 2 chroma values, $D_{H}$ depends on the arithmetic mean value of both chroma and hue values of compared color 1 and color 2 , and $R_{T}$ is a correlation value between one factor that depends on the arithmetic mean value of compared color 1 and color 2 chroma values, and another factor that depends on the arithmetic mean value of compared color 1 and color 2 hue values. All the chroma and hue values are calculated in CIELAB color space.

In 1994, CIE proposed a new color difference called CIE94. ${ }^{10}$ The CIE94 color difference equation is given by Equation 9 .

$$
\Delta E_{94}^{*}=\sqrt{\left(\frac{\Delta L^{*}}{k_{L} S_{L}}\right)^{2}+\left(\frac{\Delta C_{a b}^{*}}{k_{C} S_{C}}\right)^{2}+\left(\frac{\Delta H_{a b}^{*}}{k_{H} S_{H}}\right)^{2}}
$$

where $S_{L}=1, S_{C}$ and $S_{H}$ depends on $C_{a b}^{*}$ of the standard. $k_{L}=k_{C}=k_{h}=1$ for reference conditions.

Recent CIE color-difference activities by TC1-47 will likely result in a new color difference equation called CIE2000 to be recommended. ${ }^{11}$ This formula is similar to BFD color difference in form with more consistent trends in lightness and hue-angle dependencies. The CIE2000 Color difference shown in Equation 10 is the last 
formulation of corrections designed to improve the earlier color difference equations based on pre-determined color data sets.

$$
\Delta E_{2000}=\sqrt{\left(\frac{\Delta L^{*}}{K_{L} S_{L}}\right)^{2}+\left(\frac{\Delta C^{\prime}}{K_{C} S_{C}}\right)^{2}+\left(\frac{\Delta H^{\prime}}{K_{H} S_{H}}\right)^{2}+R_{T}\left(\frac{\Delta C^{\prime} \Delta H^{\prime}}{S_{C} S_{H}}\right)}
$$

where $C^{\prime}=\sqrt{a^{\prime}+b^{* 2}}$

$$
\begin{aligned}
& h^{\prime}=\tan ^{-1}\left(b^{*} / a^{\prime}\right) \\
& \Delta H^{\prime}=2 \sin \left(\frac{\Delta h^{\prime}}{2}\right) \sqrt{C_{1}^{\prime} C_{2}^{\prime}} \\
& a^{\prime} \text { depends on } a^{*} \text { and } C^{*}, \\
& R_{T} \text { depends on the arithmetic means of both } h^{\prime} \text { and } C^{\prime} \\
& L^{*}, a^{*}, b^{*}, C^{*} \text { are calculated in CIELAB, } C_{1}^{\prime} \text { and } C_{2}^{\prime} \text { are the } C^{\prime} \text { values for color }
\end{aligned}
$$

1 and color 2, respectively. More details of the CIE2000 Color difference will be available when the TC1-47 committee recommends it.

More details of the history and development of color difference formulae can be found in Reference 12. These color difference equations were developed using colored patches data sets in whole visible range, not for images containing complex scenes (complex images).

In the last decade, many researchers are concentrating efforts to derive color difference formulation for complex images. Among the most interesting results, we should mention the color image fidelity metric S-CIELAB.${ }^{13}$ S-CIELAB is an extension 
of the CIELAB $\Delta E_{a b}$ color difference formula. The extension is in the form of a spatial pre-processing step that incorporates the pattern-color sensitivity measurements. In another research, Tremeau et al. proposed a local color correlation measure for color image comparison based on characteristics of human visual perception. ${ }^{14}$

Although color difference could be defined locally, it is not an easy task to derive a simple formula for color difference because the color difference perceptibility depends on the contents of the images. ${ }^{15}$

In applications for three-dimensional gamut-mapping techniques of computer generated images, Katoh and al. customized the color difference in CIELAB color space using psychophysical techniques. ${ }^{16}$ The weighted formula is shown in Equation 11.

$$
\Delta E_{K}=\sqrt{\left(\frac{\Delta L^{*}}{K_{L}}\right)^{2}+\left(\frac{\Delta C_{a b}{ }^{*}}{K_{C}}\right)^{2}+\left(\frac{\Delta H_{a b}{ }^{*}}{K_{H}}\right)^{2}}
$$

where $K_{L}, K_{C}$, and $K_{H}$ are the weighting coefficients for lightness, chroma and hue respectively.

Another work that should be mentioned has been performed by Gibson, ${ }^{17}$ in which colorimetric tolerances in terms of lightness, chroma and hue are evaluated for various images (for instance a portrait, a natural scene and a man-made scene). This work extends to different monitor technologies and hardcopy previous colorimetric tolerance experiment for digital images performed by Stokes. ${ }^{18}$ 
With the advent and proliferation of digital imaging, the scientific community recognizes that there is an urgent need for recommendations to derive and report color differences for images as opposed to single pairs of color patches. The TC8-02 Colour Difference Evaluation in Images technical committee ${ }^{19}$ has been developing guidelines and among their recommendation in deriving a color difference average equation, the TC8-02 recommends that the correlation between the color coordinates (for instance $\Delta L^{*}, \Delta a^{*}$, and $\Delta b^{*}$ ) should be considered and reported, assuming that they have an approximately Gaussian distribution.

The $\operatorname{CMC}(l: c), \operatorname{BFD}(l: c)$, CIE94 equations and the CIE2000 equation, that is likely to appear soon, take into account the correlation between hue and chroma in the estimation of weighting functions. These color difference equations were derived using colored patches and not complex images used in practical applications. Colored patch sets such as the Munsell Colors Set allowed researchers to derive color difference equations precisely in controlled environment. However when dealing with complex images a model that is more flexible and general is necessary because the experimenter could not fine-tune the equations as obtained for colored patches. One can envision a more general metric that can be easily derived based on images using perceptually correlated parameters such as lightness, chroma and hue angle. A candidate for this metric is the Mahalanobis distance that consider the correlation between each attribute. 
In this paper, a color difference is defined by Mahalanobis distance ${ }^{20}$ using covariance matrix of differences of metric lightness, chroma and hue angle between two images. The covariance matrix is obtained by psychophysical experiments changing the metric lightness, chroma and hue angle of the images. These experiments provide us some preliminary analysis of the potential information we can extract from the proposed perceptual color difference metric.

\section{Proposal of a perceptual color difference metric based on Mahalanobis distance}

The Mahalanobis distance shown in Eq. 12, commonly used in pattern recognition analysis, makes uniform the influence of the distribution of each attribute $X 1, X 2, \ldots, X \mathrm{n}$ considering the correlation between each term.

$$
\Delta d=\sqrt{\left[\begin{array}{llll}
\Delta X 1 & \Delta X 2 & \cdots & \Delta X n
\end{array}\left[\begin{array}{cccc}
\sigma_{X 1, X 1} & \sigma_{X 1 X 2} & \cdots & \sigma_{X 1 X n} \\
\sigma_{X 2 X 1} & \sigma_{X 2, X 2} & \cdots & \sigma_{X 2 X n} \\
\vdots & \vdots & \ddots & \vdots \\
\sigma_{X n X 1} & \sigma_{X n X 2} & \cdots & \sigma_{X n, X n}
\end{array}\right]^{-1}\left[\begin{array}{c}
\Delta X 1 \\
\Delta X 2 \\
\vdots \\
\Delta X n
\end{array}\right]\right.}
$$

$\sigma_{\mathrm{Xi}, X \mathrm{i}}$ is the variance of the attribute $X \mathrm{i}$, and $\sigma_{\mathrm{Xi}, \mathrm{Xj}_{\mathrm{j}}}$ is the covariance between attributes $X \mathrm{i}$ and $X \mathbf{j}$.

The variance-covariance matrix of the Eq. 12 can be derived using a similar technique to the method used to derive Brown-MacAdam ellipsoids. ${ }^{21}$ In Brown and MacAdam experiment they used a method in which the observer could vary the amounts 
of all three mixtures of red, green and blue primaries in the variable stimulus and match a fixed stimulus. They computed the variances using Eqs. 13.

$$
\begin{aligned}
& \operatorname{var}(R)=\sigma_{R, R}=\frac{1}{n-1} \sum_{i=1}^{n}\left(R_{i}-R_{o}\right)^{2} \\
& \operatorname{var}(G)=\sigma_{G, G}=\frac{1}{n-1} \sum_{i=1}^{n}\left(G_{i}-G_{o}\right)^{2} \\
& \operatorname{var}(B)=\sigma_{B, B}=\frac{1}{n-1} \sum_{i=1}^{n}\left(B_{i}-B_{o}\right)^{2}
\end{aligned}
$$

where $R_{i}, G_{i}, B_{i}$, are the values of the $i$ th match $(i=1,2,3, \ldots, \mathrm{n})$ and $R_{o}, G_{o}, B_{o}$ are the values of the mean color given by Eq. 14 .

$$
T_{o}=\frac{1}{n} \sum_{i=1}^{n} T_{i}
$$

where $T=(R, G, B)$.

The covariances can be calculated as shown in Eqs 15.

$$
\begin{aligned}
& \sigma_{R, G}=\operatorname{cov}(R, G)=\sigma_{G, R}=\operatorname{cov}(G, R)=\frac{1}{n-1} \sum_{i=1}^{n}\left(R_{i}-R_{o}\right)\left(G_{i}-G_{o}\right) \\
& \sigma_{G, B}=\operatorname{cov}(G, B)=\sigma_{B, G}=\operatorname{cov}(B, G)=\frac{1}{n-1} \sum_{i=1}^{n}\left(G_{i}-G_{o}\right)\left(B_{i}-B_{o}\right) \\
& \sigma_{R, B}=\operatorname{cov}(R, B)=\sigma_{B, R}=\operatorname{cov}(B, R)=\frac{1}{n-1} \sum_{i=1}^{n}\left(R_{i}-R_{o}\right)\left(B_{i}-B_{o}\right)
\end{aligned}
$$

The calculated variances and covariances are used to derive the variancecovariance matrix given by Eq. 16 . 


$$
\boldsymbol{M}=\left[\begin{array}{lll}
\sigma_{R, R} & \sigma_{R, G} & \sigma_{R, B} \\
\sigma_{G, R} & \sigma_{G, G} & \sigma_{G, B} \\
\sigma_{B, R} & \sigma_{B, G} & \sigma_{B, B}
\end{array}\right]
$$

The elements of the matrix $\boldsymbol{M}^{-1}$, the inverse of matrix $\boldsymbol{M}$, are used to derive the BrownMacAdam ellipsoids.

Color attributes such as metric lightness, chroma and hue angle can be more related to human perception instead of using $R, G, B$. The Mahalanobis distance can be applied in a color space using metric lightness, chroma and hue angle as follows;

$$
\begin{aligned}
& \Delta d=\sqrt{\left[\begin{array}{lll}
\Delta L & \Delta C & \Delta h
\end{array}\right]\left[\begin{array}{lll}
\sigma_{L L} & \sigma_{L C} & \sigma_{L h} \\
\sigma_{C L} & \sigma_{C C} & \sigma_{C h} \\
\sigma_{h L} & \sigma_{h L} & \sigma_{h h}
\end{array}\right]^{-1}\left[\begin{array}{l}
\Delta L \\
\Delta C \\
\Delta h
\end{array}\right]} \\
& \Delta d=\sqrt{\left[\begin{array}{lll}
\Delta L & \Delta C & \Delta h
\end{array}\right]\left[\begin{array}{lll}
W_{L L} & W_{L C} & W_{L h} \\
W_{C L} & W_{C C} & W_{C h} \\
W_{h L} & W_{h L} & W_{h h}
\end{array}\right]\left[\begin{array}{l}
\Delta L \\
\Delta C \\
\Delta h
\end{array}\right]}
\end{aligned}
$$

where $\sigma_{L L}, \sigma_{C C}, \sigma_{h h}$ are the variances of metric lightness, chroma, hue angle, respectively. $\Delta L, \Delta C$, and $\Delta h$ are respectively difference of the metric lightness, chroma and hue angle difference between two images, for instance the original and the reproduction. On the other hand, $\sigma_{L C}\left(\sigma_{C L}\right), \sigma_{L h}\left(\sigma_{h L}\right), \sigma_{C h}\left(\sigma_{h C}\right)$ are the covariances between metric lightness and chroma, and lightness and hue angle, and chroma and hue angle, respectively. The variance-covariance matrix can be easily derived using threedimensional threshold of color-difference perceptibility as shown above..$^{21-23}$ The terms 
of the matrix in Eq. 18 give clues of how sensitive our perception is for a certain image in terms of metric lightness, chroma and hue angle.

Rewriting Eq. 18 considering that $W_{L C}=W_{C L}, W_{L h}=W_{h L}$, and $W_{C h}=W_{h C}$ we have Eq. 19.

$$
\Delta d=\sqrt{W_{L L} \Delta L^{2}+W_{C C} \Delta C^{2}+W_{h h} \Delta h^{2}+2 W_{L C} \Delta L \Delta C+2 W_{L h} \Delta L \Delta h+2 W_{C h} \Delta C \Delta h}
$$

The term $W_{L L}$ of the matrix has influence on the perceptual metric lightness sensitivity. The term $W_{C C}$ of the matrix has influence on the perceptual chroma sensitivity. The term $W_{h h}$ of the matrix has influence on the hue angle sensitivity. The term $W_{L C}\left(W_{C L}\right)$ of the matrix has influence on the correlation between metric lightness and chroma. The term $W_{L h}\left(W_{h L}\right)$ of the matrix has influence on the correlation between metric lightness and hue angle. The term $W_{C h}\left(W_{h c}\right)$ of the matrix has influence on the correlation between chroma and hue angle. If there is no correlation between metric lightness, chroma and hue angle, the distance is reduced to a weighted Euclidian distance given the Eq. 20.

$$
\Delta d=\sqrt{W_{L L} \Delta L^{2}+W_{C C} \Delta C^{2}+W_{h h} \Delta h^{2}}
$$

Comparing the Eq. 20 with the CIE94 color difference equation given by Eq. 9 we can derive the CIE94 color difference equation from the simplified Mahalanobis perceptual difference shown in Eq. 21 where $W_{L L}=\left(\frac{1}{k_{L} s_{L}}\right)^{2}, W_{C C}=\left(\frac{1}{k_{C} s_{C}}\right)^{2}$ and $W_{h h}=\left(\frac{1}{k_{h} s_{h}}\right)^{2}$ 
The weights in the simplified Eq. 20 can also be correlated with the weights in the Eq. 11 proposed by Katoh et al.

One possible application for this perceptual distance is the evaluation of gamutmapping techniques. The gamut-mapping technique that provides the shortest perceptual Mahalanobis distance is considered as the best technique. Ito et al. also has successfully used Mahalanobis distance between out-of-gamut color and a color on the gamut boundary for clipping method ${ }^{24}$ based on this metric that we derived.

\section{Psychophysical experiments}

Six images were used in these experiments consisting of four electronic endoscope stomach images and two natural scenes depicting a portrait and a flower. Although not very conventional scenes, the electronic endoscope images were chosen because we already have the results of an earlier psychophysical experiments using such images. ${ }^{25-27}$ In our experiments CIELUV color space was employed in the calculations of the metric lightness, chroma and hue angle. CIELUV color space was chosen to allow us to compare the results of the endoscope images to a previous psychophysical experiments performed by Hara et al. using physicians as subjects. ${ }^{27}$

For each of the images, the original image (reference) and a modified image was displayed side-by-side on the center of a calibrated Nanao FlexScan 56T CRT display, in a dark environment. The monitor was adjusted for luminance $93.3 \mathrm{~cd} / \mathrm{m}^{2}$, D65 white 
point and maximum contrast. We changed randomly the relative position on the CRT display of the reference and the modified images. The color distribution of the images in $\mathrm{L} * \mathrm{xa}^{*}$ and $\mathrm{a} * \mathrm{xb} *$ diagrams (D65, 2 degree observer) are shown from Figures 1a to 1f. Table I shows the average $\mathrm{L}^{*}, \mathrm{a}^{*}, \mathrm{~b}^{*}$, and $\mathrm{C}^{*}$ values for each image.

A preliminary and exhaustive experiment was performed with one subject to select appropriate values and intervals for changing the images. We do not want to use too many steps since we are working with three dimensions simultaneously. But at the same time we need sufficient data to calculate the variance-covariance matrix. As a result the metric lightness of the image was changed by $-4,-2,0,2,4$ units, the chroma was changed by $-6,-3,0,3,6$ units, and the hue angle was changed by $-2,-1,0,1,2$ degrees depart from the original image. Every possible combination of these color attributes was prepared resulting in 125 images for each endoscope image. Ten observers (students at Chiba University) were asked to watch each pair on CRT and asked to answer if the images are noticeable different or not.

Table II shows the displacements of the color attributes from the original image that produced non-perceptible difference for one of the electronic endoscope image (the electronic endoscope image 4 ). Figures $2 \mathrm{a}, 2 \mathrm{~b}$ and $2 \mathrm{c}$ show respectively, the plots of the correlation between metric lightness and chroma, chroma and hue angle and metric lightness and hue angle. From the experimental data we calculated the variancecovariance matrix that is used in the perceptual color difference, as shown in Eq. 21. 


$$
\Delta d=\sqrt{\left[\begin{array}{lll}
\Delta L & \Delta C & \Delta h
\end{array}\right]\left[\begin{array}{ccc}
1.48 & 4.22 & -0.72 \\
4.22 & 0.0375 & -2.00 \\
-0.72 & -2.00 & 0.35
\end{array}\right]^{-1}\left[\begin{array}{c}
\Delta L \\
\Delta C \\
\Delta h
\end{array}\right]}
$$

The covariances between metric lightness and chroma, and lightness and hue angle, and chroma and hue angle, respectively $\sigma_{L C}\left(\sigma_{C L}\right)=4.22, \sigma_{L h}\left(\sigma_{h L}\right)=-0.72$, $\sigma_{C h}\left(\sigma_{h C}\right)=-2.00$ are in accord to the ellipsis inclinations in the plots of Figure 2.

Rewriting the perceptual distance metric we have Eq. 22.

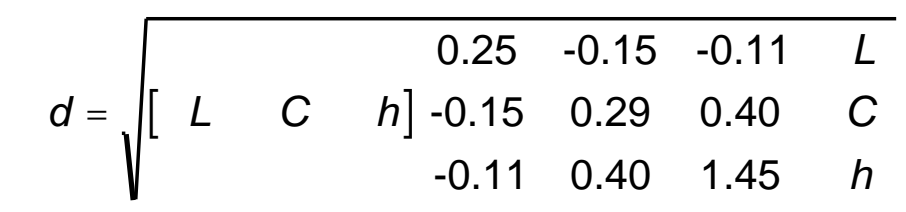

From equation 22, it is possible to see that in terms of perceptual tolerance for this particular electronic endoscope image, we are more sensitive to unit changes in hue angle than changes in chroma or metric lightness. The negative value of the correlation between metric lightness and chroma tells us that if we change metric lightness and chroma of the image simultaneously it is possible to minimize the perceptual color difference. The same analysis applies for the correlation between metric lightness and hue angle.

\section{Results and Discussion}

The results of the experiment using electronic endoscope images were used to derive the covariance matrix using statistical methods. The resultant inverse of variancecovariance matrices are shown in Fig. 3a, 3b, 3c, and 3d with the images. We can see 
the characteristics of the electronic endoscope images from these matrices. First, the element $W_{h h}$ is larger than any other elements in all the matrices. This indicates that hue angle should be maintained unchanged in the electronic endoscope images to keep the perceived color of the images. Second, the elements $W_{L C}\left(W_{C L}\right)$ and $W_{L h}\left(W_{h L}\right)$ are negative. This indicates that the lightness and chroma, lightness and hue angle should be increased or decreased simultaneously in direction of the same sign to minimize the difference in perception between the images. Third, the element $W_{C h}\left(\mathrm{~W}_{h C}\right)$ is positive. This indicates that the lightness and hue angle should be increased or decreased in direction of the opposite sign to minimize the perceived difference of the images.

Another interesting result is the fact that images with more details and therefore higher spatial frequency, such as the gastritis of the electronic endoscope image 1 (Figure 3a) and the polyp of the electronic endoscope image 3 (Figure 3c) presented larger $W_{h h}$ value than a more uniform image such as the electronic endoscope image 2 (Figure 3b) showing evidences that images with more details are more sensitive to changes in hue angle. This result correlates well with the color distribution. From Figures $1 \mathrm{a}, 1 \mathrm{~b}$ and $1 \mathrm{c}$ it is possible to see that the electronic endoscope images 1 and 3 present a much wider color distribution than the electronic endoscope image 2.

Hara and coworkers carried out a psychophysical experiment ${ }^{27}$ with collaboration of five medical doctors experienced in diagnosis based on endoscope 
images. They changed the metric lightness, chroma and hue angle in order to reproduce a preferred endoscope image on CRT. In this experiment, they only examined variance of metric lightness, chroma, hue angle and covariance between metric chroma and hue angle. Their results are summarized in Table III. Although we can not calculate the perceptual color difference $\Delta d$ it is possible to observe that the variance of metric chroma was greater than that of lightness, the variance of lightness was greater than that of hue angle, and covariance between chroma and hue angle have a negative value like the results of our experiments. Here, we note that the matrices of Figure 3 should be inverted to get the corresponding covariance matrices for each image. From these results we assume that our experimental results by students is appropriate in comparison with the results by experts in medicine.

Figure 4 shows the resultant inverse covariance with the corresponding images for the natural scene images. The matrices for natural scenes were fairly different from those for the electronic endoscope images. Particularly, the sign of non-diagonal elements is different between natural scenes and electronic endoscope images.

We believe that different images from the same class of images (portrait, landscape, etc...) will have different values for the terms in the matrix of the Mahalanobis distance. However, the relative values and the presence of positive and negative terms will characterize the images in each class. For example, for the endoscopic images every image presented negative terms for the correlation between 
metric lightness and chroma, and the correlation between metric lightness and hue angle. This analysis can give us clues of how we can minimize the perceptual distance. In order to compare the proposed perceptual color difference equation with $\Delta E^{*}{ }_{94}$ we computed the weights of the Eq. 9 for each image using the average CIELAB values shown in Table I, D65 illuminant and 2 degree observer. Table IV shows the calculated $\Delta E^{*}{ }_{94}$ weights and its corresponding weights in the reduced Mahalanobis distance perceptual color difference without covariance factors. Since we had to use CIELUV to calculate the reduced Mahalanobis distance perceptual color difference parameters, we can not compare quantitatively the parameters to the $\Delta E^{*}{ }_{94}$ weights unfortunately. However, from the quantitative point of view the $\Delta E^{*}{ }_{94}$ weights presented relative values that are completely different from what we obtained using the reduced Mahalanobis distance perceptual color difference.

Although more experiments are needed to reach conclusive results, we believe that it is possible to customize this metric according to different classification of complex images and probably the contents of the image, and not only the average color, is related to the variance-covariance matrix. 


\section{Conclusion}

A Mahalanobis distance for color difference was proposed as a general perceptual color difference formula. This perceptual distance is calculated by using covariance matrix for the differences of metric lightness, chroma and hue angle, and indicates how the perceived color of reproduced image is affected by changes in the attributes of color. This perceptual color difference provides a general way to apply further analysis of the color difference in complex images. It also has the advantage to simplify the color difference equation. The color difference is not the only one aspect of many differences between original image and processed image that can affect the quality of the reproduction. For example, the contrast of the images is one of the important aspects to evaluate the images. In future experiments, we also should consider the spatial information ${ }^{13,28}$ to improve the evaluation techniques furthermore and evaluate the influence of the display type (CRT, flat panel) or difference between hardcopy and softcopy on the calculations of this perceptual color difference for complex images.

\section{References}

1) CIE Publication No. 15.2, Colorimetry, $2^{\text {nd }}$ ed., Commission Internationale de l’Éclairage, Vienna, Austria, (1986).

2) K. McLaren, "An introduction to instrumental shade passing and sorting and a review of recent developments," J. Soc. Dyers Colour. 92(9), 317-326 (1976). 
3) R. McDonald, "The effect of non-uniformity in the ANLAB color space on the interpretation of visual colour differences," J. Soc. Dyers Colour. 90(6), 189-198 (1974).

4) R. McDonald, "Industrial pass/fail colour matching. Part III - Development of pass/fail formula for use with instrumental measurement of colour difference," J. Soc. Dyers Colour. 96(9), 486-495 (1980).

5) D. Nickerson, "Munsell renotations used to study color spaces of Hunter and Adams," J. Opt. Soc. Am. 40(2), 85-88 (1950).

6) F. J. J. Clarke, R. McDonald, and B. Rigg, "Modification to the JPC 79 colourdifference formula," J. Soc. Dyers Colour. 100(4), 128-132 (1984).

7) R. McDonald, "Acceptability and Perceptibility decisions using the CMC color difference formula," Textiles chemist and colorist. 20(6), 31-36(1988).

8) M. R. Luo and B. Rigg, "BFD (l:c) colour-difference formula. Part 1 - Development of the formula," J. Soc. Dyers Colour. 103(2), 86-94 (1987).

9) M. R. Luo and B. Rigg, "BFD (l:c) colour-difference formula. Part 2 - Performance of the formula," J. Soc. Dyers Colour. 103(3), 126-132 (1987).

10) CIE, Publication No. 116, Industrial Colour-Difference Evaluation, Commission Internationale de l'Éclairage, Vienna, Austria, (1995).

11) CIE, TC1-47, Hue and Lightness Correction to Industrial Colour Difference Evaluation, Tentative CIE2000 Color Difference Equation, April, 2000. 
12) R. S. Berns, Billmeyer and Saltzman principles of color technology, $3^{\text {rd }}$ ed., John Wiley \& Sons, Inc., New York, NY, 2000, pp. 107-122.

13) X. M. Zhang and B. A. Wandell, A spatial extension to CIELAB for digital color images reproduction, In Society for Information Display Symposium, San Diego, CA, 1996.

14) A. Tremeau, V. Pugnet, E. Dinet and B. Laget, A local color correlation measure for color image comparison, in IS\&T/SID Fourth Color Imaging Conference: Color Science, Systems, and Applications, IS\&T, Springfield, VA, 1996, pp. 119-122.

15) S, P. Farnand, The effect of image content on color difference perceptibility, in IS\&T/SID Third Color Imaging Conference: Color Science, Systems, and Applications, IS\&T, Springfield, VA, 1995, pp. 101-104.

16) N. Katoh, M. Ito, Gamut mapping for computer generated images (2), in $I S \& T / S I D$ Fourth Color Imaging Conference: Color Science, Systems, and Applications, IS\&T, Springfield, VA, 1996, pp. 126-129.

17) J. E. Gibson, Colorimetric Tolerances of various digital image displays, M. Sc. Thesis, 2000, to appear.

18) M. Stokes, Colorimetric tolerances of digital images, M. Sc. Thesis, Rochester Institute of Technology, Rochester, NY, 1991.

19) CIE, TC8-02, Methods to derive colour difference for images, Draft report version 0.3, http://www.colour.org/tc8-02/cietc802v03.pdf, June, 1999. 
20) R. O. Duda and P. E. Hart: Pattern Classification and scene analysis, John Wiley \& Sons, 1973, p.24.

21) G. Wyszecki and W. S. Stiles: Color Science: Concepts and Methods, Quantitative data and Formulae, 2nd ed., John Wiley \& Sons, 1982.

22) K. Witt, Three-Dimension Threshold of Color-Difference Perceptibility in Painted Samples: Variability in Painted Samples: Variability of Observers in Four CIE Color Regions, Col. Res. Appl. 12, 128-134 (1987).

23) J. Uroz, Colour Difference Perceptibility for Large-Size Printed Images, M. Sc. Thesis, University of Derby, Derby, 1999.

24) M. Ito, N. Katoh, Three-dimensional gamut mapping using various color difference formulae and color spaces, in IS\&T/SPIE Conference on color imaging: deviceindependent Color, Color Hardcopy, and Graphic Arts IV, San Jose, Proc. SPIE Vol. 3648, 1999, pp. 83-95.

25) F. H. Imai, Color reproduction of facial pattern and endoscopic image based on color appearance models, Ph.D. Thesis, Chiba University, 1996.

26) N. Tsumura, F. H. Imai, T. Saito, H. Haneishi and Y. Miyake, Color gamut mapping based on Mahalanobis distance for color reproduction of electronic endoscope image under different illuminant, in IS\&T/SID Fifth Color Imaging Conference: Color Science, Systems, and Applications, IS\&T, Springfield, VA, 1997, pp. 158-162. 
27) K. Hara, H. Haneishi, H. Yaguchi, Y. Miyake, "On the preferred color reproduction of electric endoscopic images," Proc. of 23rd Engineering Image conference, 1992, pp. 119-122, in Japanese.

28) S. Nakauchi, M. Imamura, S. Usui, Color gamut mapping by optimizing perceptual image quality, in IS\&T/SID Fourth Color Imaging Conference: Color Science, Systems, and Applications, IS\&T, Springfield, VA, 1996, pp. 63-67. 

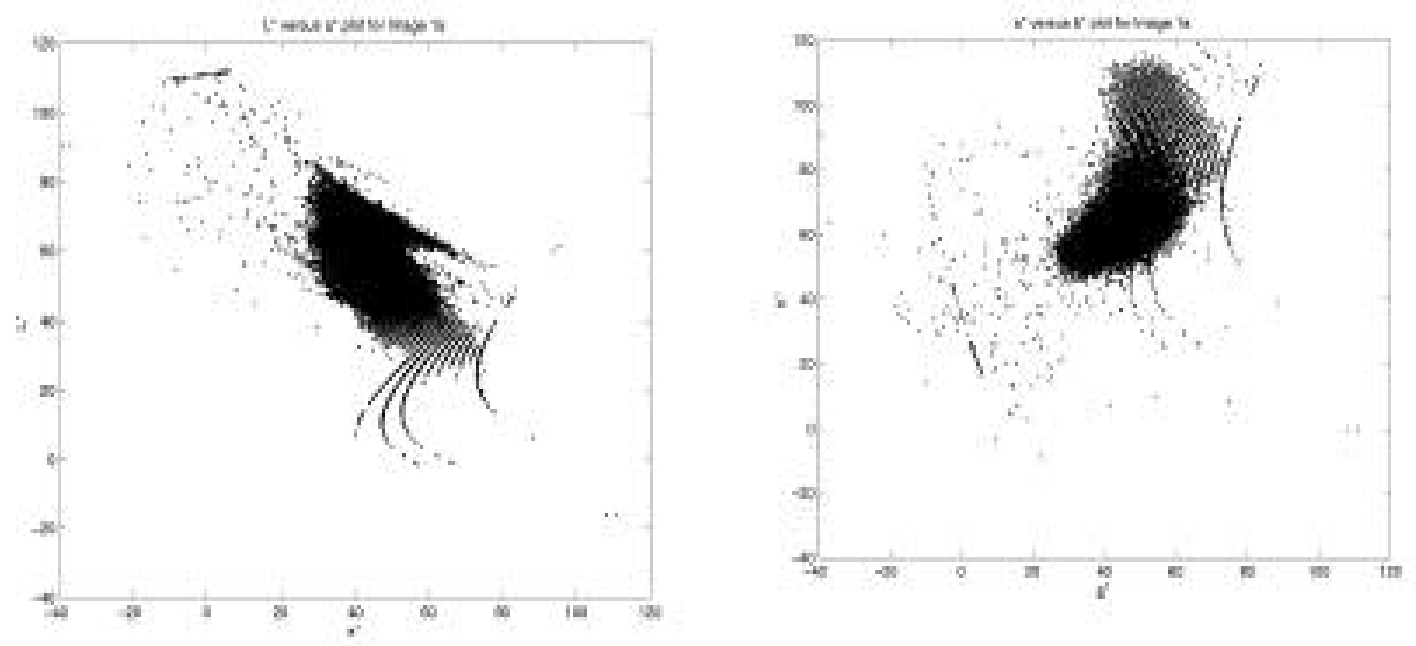

a. Electronic endoscope image 1.
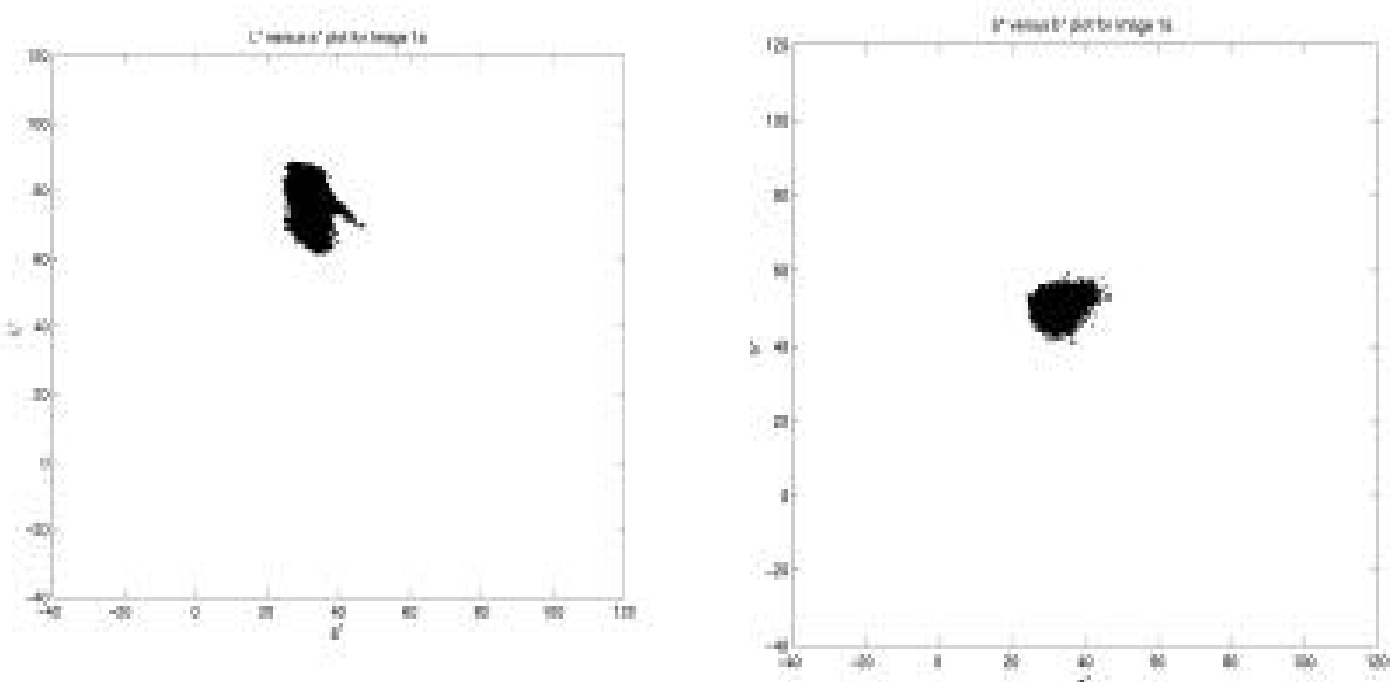

b. Electronic endoscope image 2.
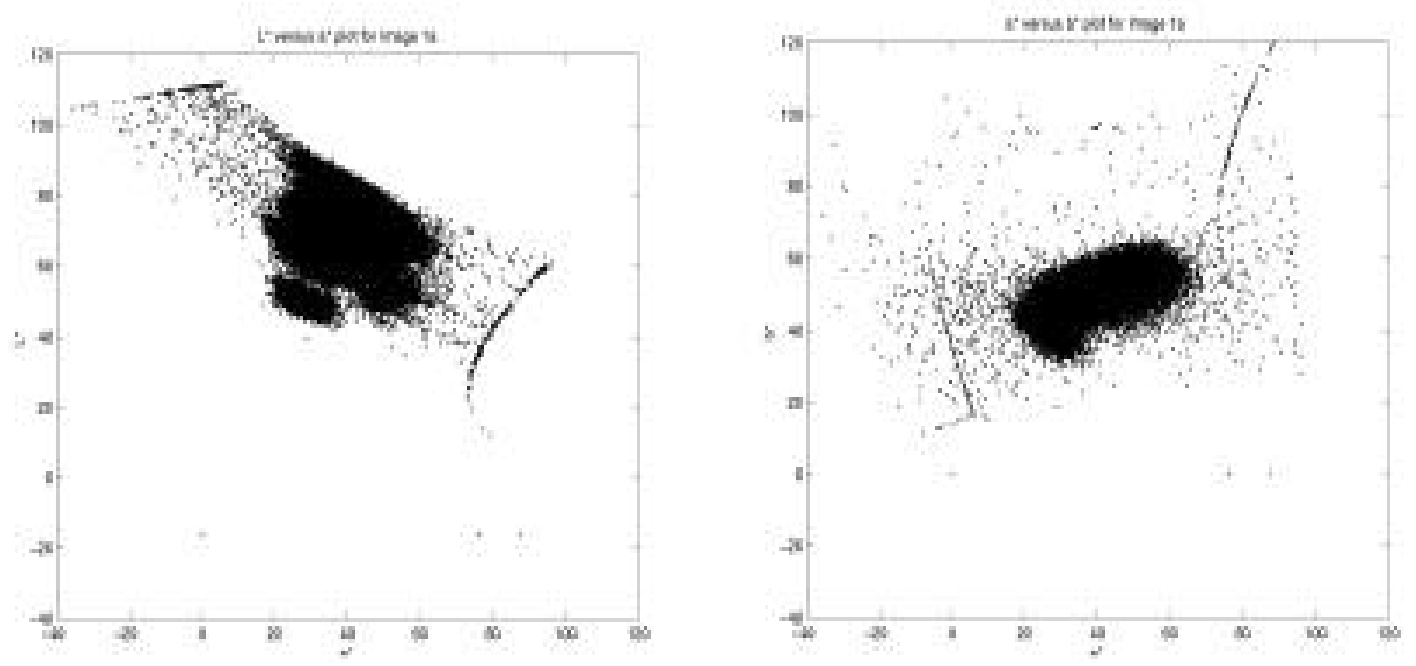

c. Electronic endoscope image 3 

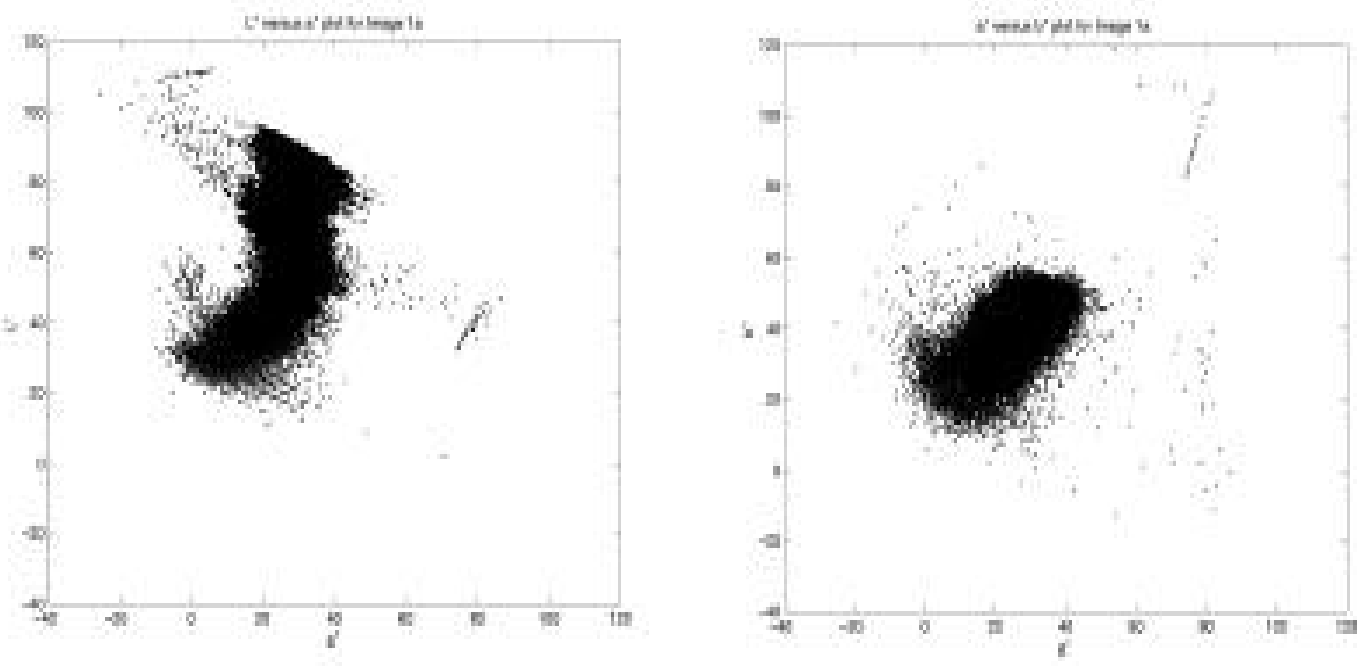

d. Electronic endoscope image 4
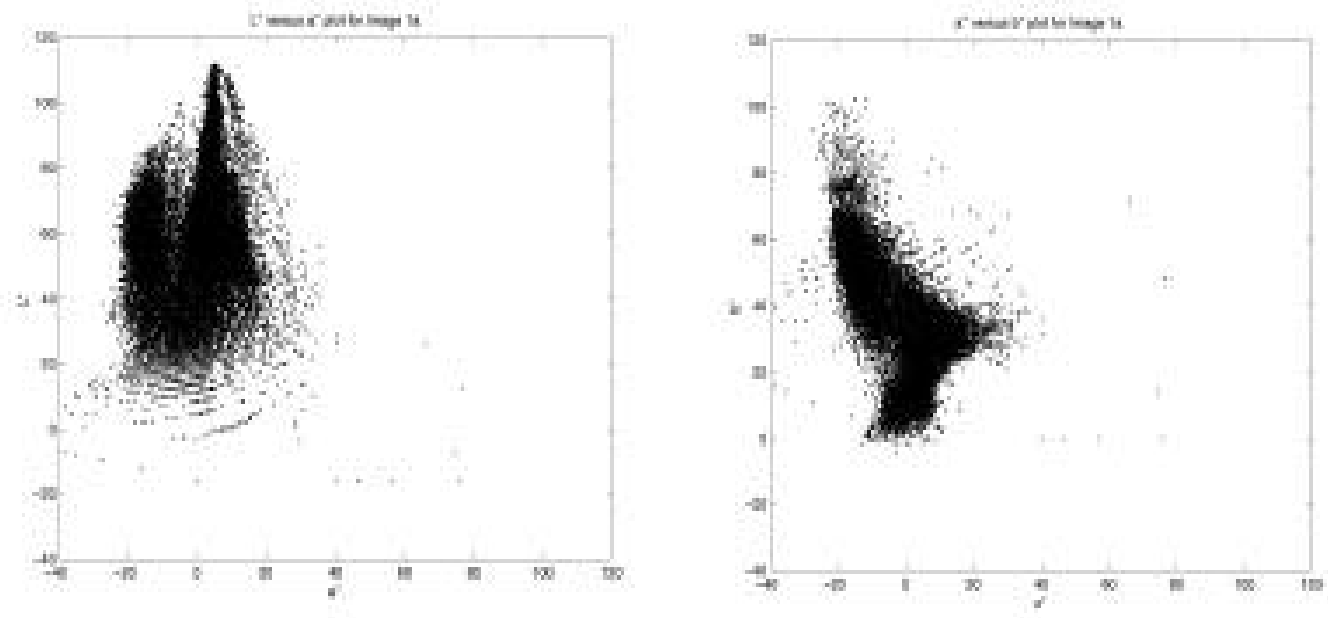

e. Potrait image
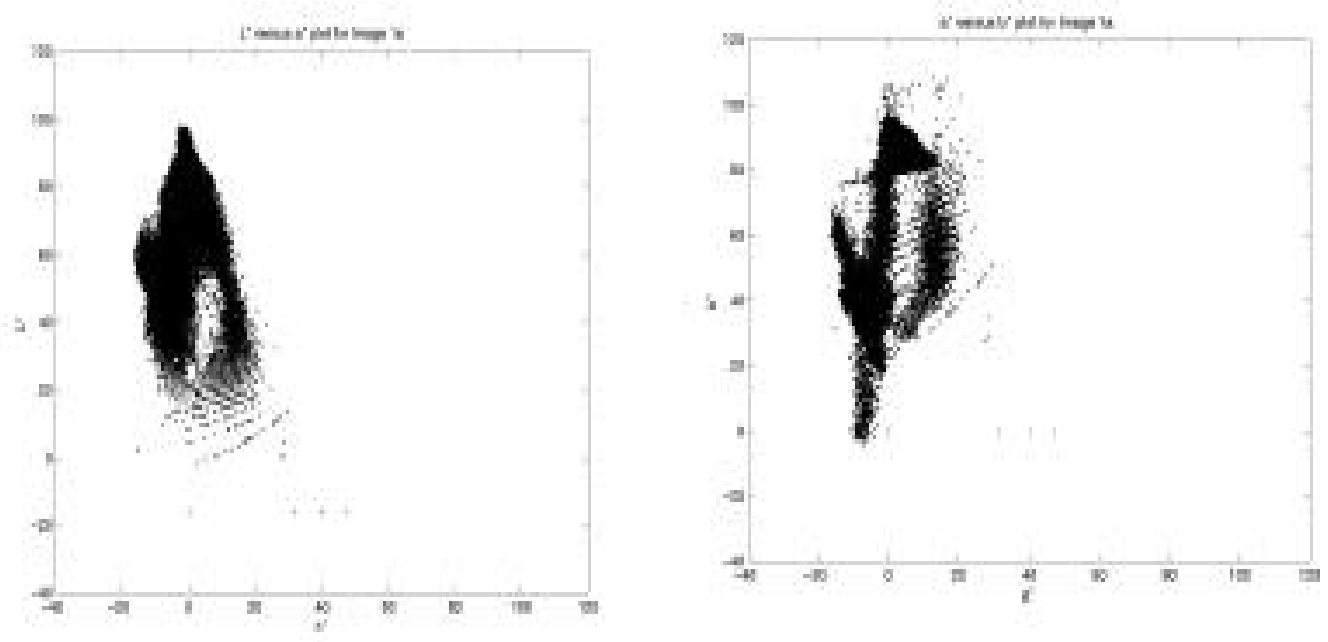

\section{f. Flower image}

Figure 1. Plots of $\mathrm{L}^{*} \mathrm{xa}^{*}$ and $\mathrm{a}^{*} \mathrm{xb}^{*}$ color distribution (D50, 2 degree observer). 


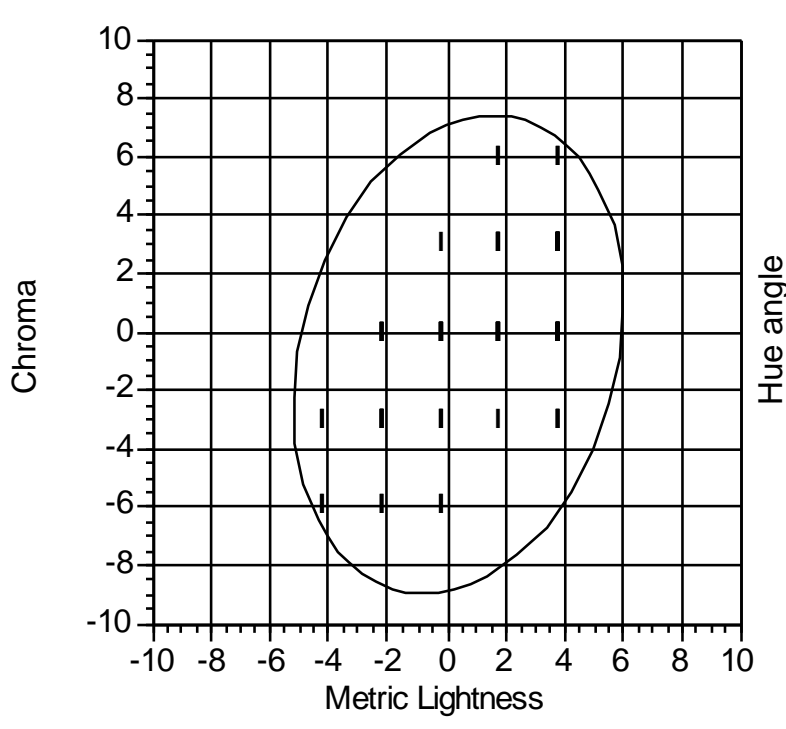

a) Metric lightness and chroma

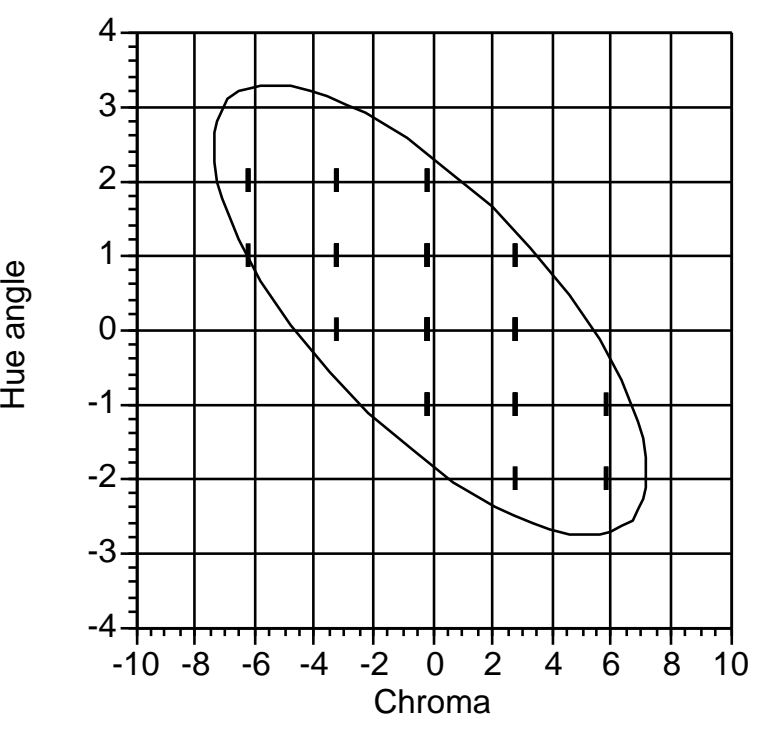

b) Chroma and hue angle

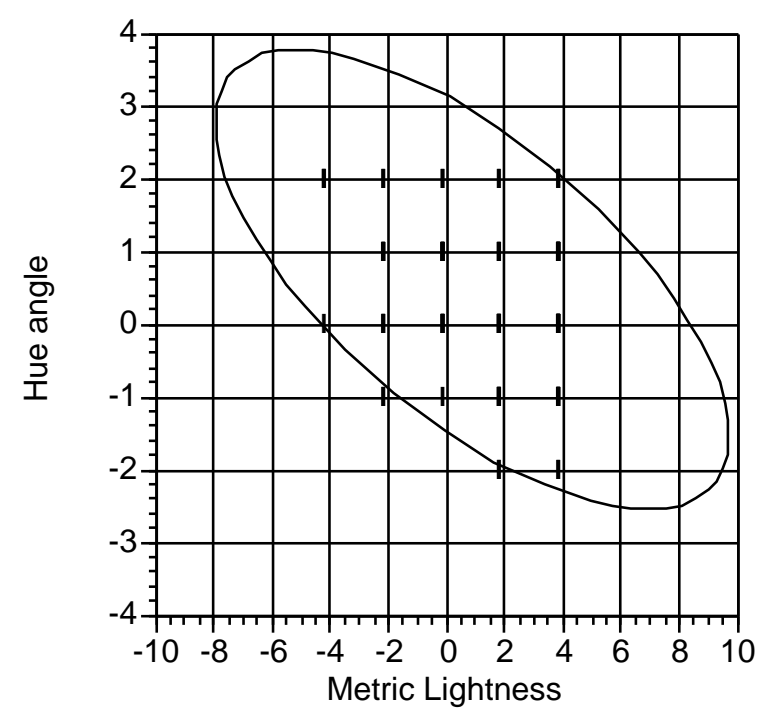

c) Metric Lightness and hue angle

Figure 2. Plots of the correlation between color attributes resulted from a psychophysical experiment using a stomach cavity image. 


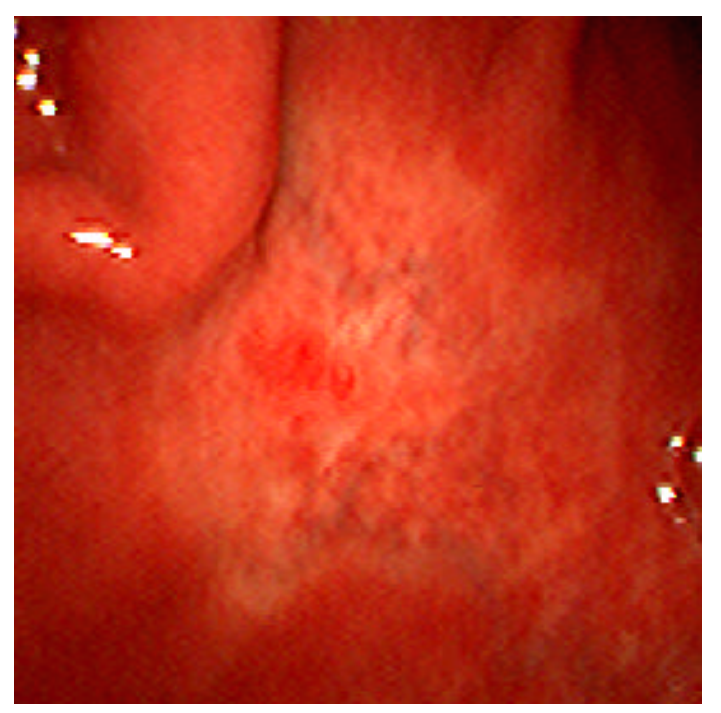

$\left[\begin{array}{ccc}0.42 & -0.15 & -0.32 \\ -0.15 & 0.21 & 0.54 \\ -0.32 & 0.54 & 2.32\end{array}\right]$

a)Electronic endoscope image 1 .

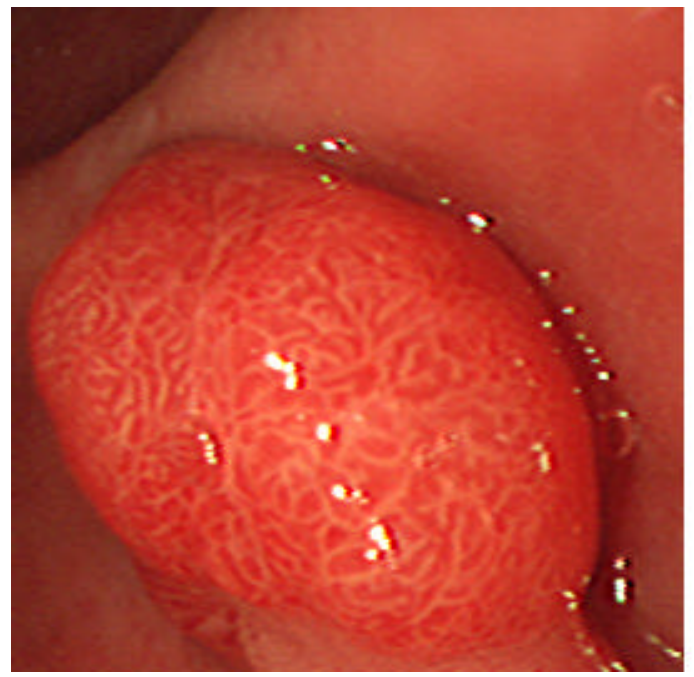

$\left[\begin{array}{ccc}0.40 & -0.16 & -0.36 \\ -0.16 & 0.25 & 0.66 \\ -0.36 & 0.66 & 2.81\end{array}\right]$

c) Electronic endoscope image 3.

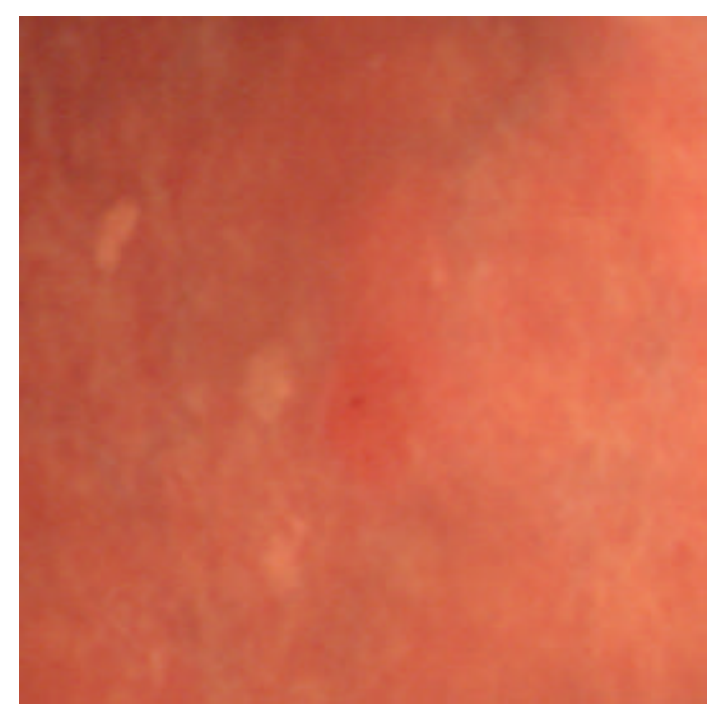

$$
\left[\begin{array}{ccc}
0.20 & -0.06 & -0.11 \\
-0.06 & 0.12 & 0.16 \\
-0.11 & 0.16 & 0.95
\end{array}\right]
$$

b) Electronic endoscope image 2.

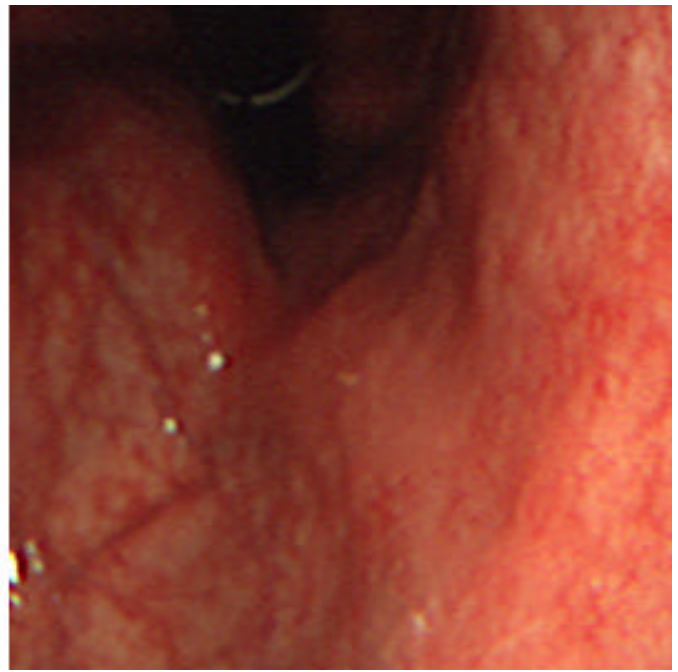

$$
\left[\begin{array}{ccc}
0.25 & -0.15 & -0.11 \\
-0.15 & 0.29 & 0.40 \\
-0.11 & 0.40 & 1.45
\end{array}\right]
$$

c) Electronic endoscope image 4 .

Figure 3. Electronic endoscope images and their respective inverse covariance matrices 


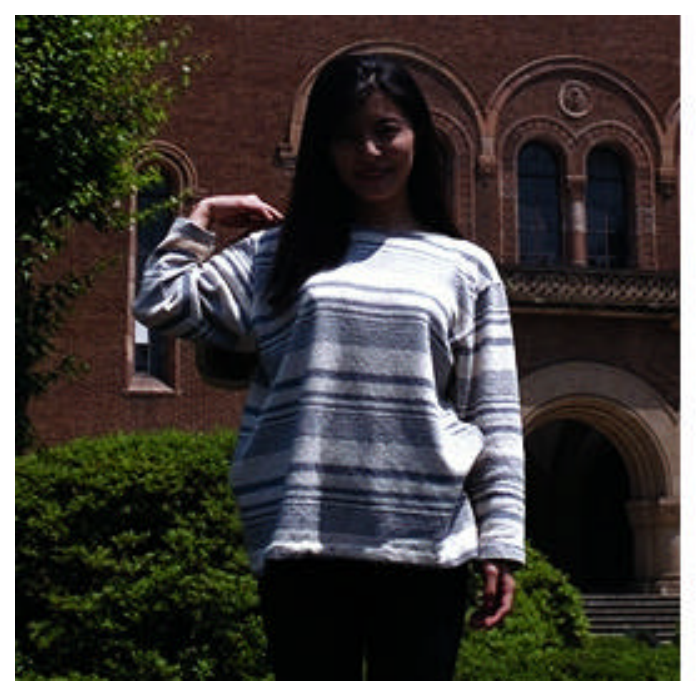

$$
\left[\begin{array}{lll}
0.51 & 0.06 & 0.18 \\
0.06 & 0.31 & 0.16 \\
0.18 & 0.16 & 0.53
\end{array}\right]
$$

a)Portrait of a girl in front of a building.

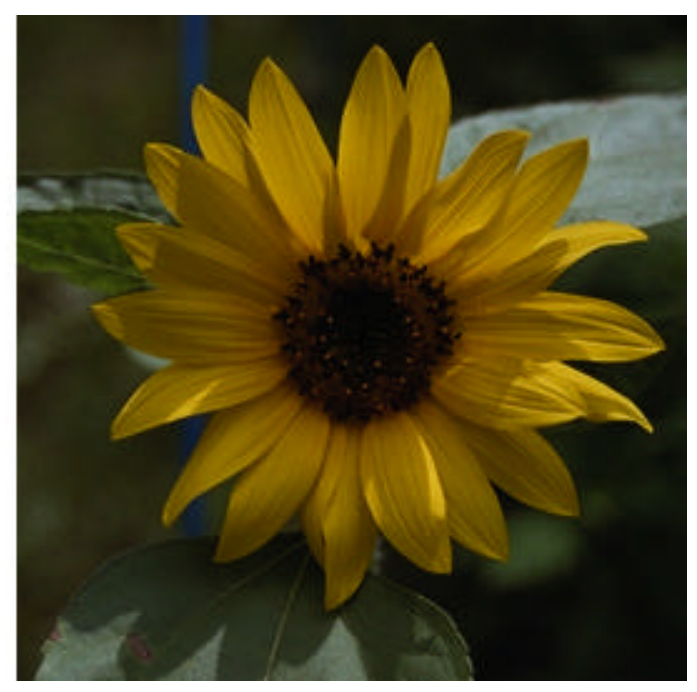

$$
\left[\begin{array}{ccc}
0.31 & -0.06 & 0.01 \\
-0.06 & 0.18 & -0.13 \\
0.01 & -0.13 & 0.93
\end{array}\right]
$$

b) Picture of a sunflower.

Figure 4. Natural scene images and their respective inverse covariance matrices 
Table I. Average CIELAB L*, a*, b*, $\mathrm{C}^{*}$ values for the images used in the experiment

\begin{tabular}{|l|c|c|c|c|}
\hline Image & $\mathrm{L}^{*}$ & $\mathrm{a}^{*}$ & $\mathrm{~b}^{*}$ & $\mathrm{C}^{*}$ \\
\hline Endoscope Image1 & 54.9 & 46.5 & $67, .3$ & 81.8 \\
\hline Endoscope Image2 & 77.3 & 32.3 & 50.6 & 60.0 \\
\hline Endoscope Image3 & 72.2 & 38.3 & 50.3 & 63.6 \\
\hline Endoscope Image4 & 64.2 & 26.0 & 41.7 & 49.1 \\
\hline Portrait Image1 & 44.7 & 0.57 & 27.7 & 27.5 \\
\hline Flower Image & 56.7 & -2.11 & 56.0 & 56.1 \\
\hline
\end{tabular}

Table II. Displacements of the color attributes (metric lightness, chroma and hue angle) that produced non-perceptible differences for the electronic endoscope image 4.

\begin{tabular}{|c|c|c|c|c|c|}
\hline $\begin{array}{c}\text { Metric } \\
\text { Lightness }\end{array}$ & Chroma & Hue & $\begin{array}{c}\text { Metric } \\
\text { Lightness }\end{array}$ & Chroma & Hue \\
\hline-4 & -6 & 2 & 2 & 0 & 1 \\
\hline-4 & -6 & 2 & 2 & 0 & 2 \\
\hline-4 & -3 & 0 & 2 & 0 & 2 \\
\hline-4 & -3 & 2 & 2 & 3 & -1 \\
\hline-2 & -6 & 1 & 2 & 3 & -1 \\
\hline-2 & -6 & 2 & 2 & 3 & 0 \\
\hline-2 & -3 & 0 & 2 & 3 & 0 \\
\hline-2 & -3 & 1 & 2 & 3 & 0 \\
\hline-2 & -3 & 1 & 2 & 6 & -2 \\
\hline-2 & -3 & 1 & 2 & 6 & -2 \\
\hline-2 & -3 & 2 & 4 & -3 & 1 \\
\hline-2 & 0 & -1 & 4 & -3 & 1 \\
\hline-2 & 0 & -1 & 4 & -3 & 2 \\
\hline-2 & 0 & 0 & 4 & 0 & -1 \\
\hline-2 & 0 & 0 & 4 & 0 & 0 \\
\hline-2 & 0 & 1 & 4 & 0 & 0 \\
\hline 0 & -6 & 1 & 4 & 0 & 0 \\
\hline 0 & -6 & 2 & 4 & 0 & 0 \\
\hline 0 & -3 & 1 & 4 & 0 & 0 \\
\hline 0 & -3 & 1 & 4 & 0 & 1 \\
\hline 0 & -3 & 2 & 4 & 0 & 1 \\
\hline 0 & 0 & -1 & 4 & 0 & 1 \\
\hline 0 & 0 & 0 & 4 & 0 & 1 \\
\hline 0 & 0 & 0 & 4 & 0 & 2 \\
\hline
\end{tabular}




\begin{tabular}{|c|c|c|c|c|c|}
\hline 0 & 0 & 0 & 4 & 0 & 2 \\
\hline 0 & 0 & 0 & 4 & 3 & -2 \\
\hline 0 & 0 & 1 & 4 & 3 & -2 \\
\hline 0 & 0 & 1 & 4 & 3 & -1 \\
\hline 0 & 0 & 1 & 4 & 3 & -1 \\
\hline 0 & 0 & 1 & 4 & 3 & -1 \\
\hline 0 & 3 & 0 & 4 & 3 & 0 \\
\hline 2 & -3 & 1 & 4 & 3 & 0 \\
\hline 2 & 0 & -1 & 4 & 3 & 0 \\
\hline 2 & 0 & -1 & 4 & 3 & 0 \\
\hline 2 & 0 & -1 & 4 & 3 & 1 \\
\hline 2 & 0 & 0 & 4 & 3 & 1 \\
\hline 2 & 0 & 0 & 4 & 3 & 1 \\
\hline 2 & 0 & 0 & 4 & 3 & 1 \\
\hline 2 & 0 & 1 & 4 & 6 & -1 \\
\hline 2 & 0 & 1 & 4 & 6 & -1 \\
\hline
\end{tabular}

Table III. Result of psychophysical experiments performed by Hara with the collaboration of physicians.

\begin{tabular}{|l|c|c|}
\hline Statistical value of color attribute & Symbol & Value \\
\hline Variance of metric lightness & $\sigma_{L L}$ & 23.6 \\
\hline Variance of metric chroma & $\sigma_{C C}$ & 34.1 \\
\hline Variance of metric hue angle & $\sigma_{h h}$ & 7.4 \\
\hline Covariance between metric chroma and hue angle & $\sigma_{C h}\left(\sigma_{h C}\right)$ & -9.0 \\
\hline
\end{tabular}

Table IV. Comparison of perceptual metric parameters with $\Delta E^{*}{ }_{94}$ parameters.

\begin{tabular}{|l|c|c|c|c|c|c|}
\hline Color Difference & \multicolumn{3}{|l|}{$\Delta E^{*}{ }_{94}(\mathrm{D} 65,2$ degree $)$} & \multicolumn{3}{l|}{ Mahalanobis distance } \\
\hline Parameter & $\left(\frac{1}{k_{L} s_{L}}\right)^{2}$ & $\left(\frac{1}{k_{C} s_{C}}\right)^{2}$ & $\left(\frac{1}{k_{h} s_{h}}\right)^{2}$ & $W_{L L}$ & $W_{C C}$ & $W_{h h}$ \\
\hline Endoscope Image1 & 1 & 0.05 & 0.20 & 0.42 & 0.21 & 2.32 \\
\hline Endoscope Image2 & 1 & 0.07 & 0.28 & 0.20 & 0.12 & 0.95 \\
\hline Endoscope Image3 & 1 & 0.07 & 0.26 & 0.40 & 0.25 & 2.81 \\
\hline Endoscope Image4 & 1 & 0.10 & 0.33 & 0.25 & 0.29 & 1.45 \\
\hline Portrait Image & 1 & 0.20 & 0.50 & 0.51 & 0.31 & 0.53 \\
\hline Flower Image & 1 & 0.08 & 0.29 & 0.31 & 0.18 & 0.93 \\
\hline
\end{tabular}

